\title{
Acessibilidade a atenção básica em um distrito sanitário de Salvador
}

\author{
Access to primary health care in a sanitary district of Salvador
}

Luciano Sepúlveda Oliveira ${ }^{1}$

Leilane Grazziela Nascimento Almeida ${ }^{1}$

Marco Aurélio Sepúlveda Oliveira ${ }^{1}$

Gabriel Brasil Gil ${ }^{1}$

Alcione Brasileiro Oliveira Cunha ${ }^{1}$

Maria Guadalupe Medina ${ }^{1}$

Rosana Aquino Guimarães Pereira ${ }^{1}$

${ }^{1}$ Instituto de Saúde Coletiva, Universidade Federal da Bahia. Rua Basílio da Gama s/n Campus Universitário,

Canela, 40110-040

Salvador BA.

83luciano@gmail.com

\begin{abstract}
The demand for and use of health services results from a set of determinants that include socioeconomic, demographic and health characteristics and organization of the supply of services. The scope of this paper is to define access to and use of the health services in two Family Health Units (FHUs) of the sanitary district of Liberdade, establishing the pattern of use and identifying factors related to socio-organizational and geographical access. A cross-sectional populationbased survey was conducted on individuals aged between 20 and 75 resident in the area covered by the two FHUs. Access to health services was analyzed based on the socio-organizational and geographic characteristics and according to the demand for medical appointments. Over $50 \%$ of respondents sought the service of FHUs and of these $78.7 \%$ reported attending the appointment. With respect to organizational access, problems were encountered, such as long waiting times for consultation. Geographical barriers were ascertained in the use of FHUs, but these did not prevent the realization of medical consultation. The use of FHUs was higher among people with lower socioeconomic status.
\end{abstract}

Key words Access to Health Services, Utilization, Family health strategy
Resumo A procura e utilização dos serviços de saúde resulta de um conjunto de determinantes que incluem características socioeconômicas, demográficas, de saúde e da organização da oferta dos serviços. Objetivo: Caracterizar a acessibilidade e a utilização de serviços de saúde em duas Unidades de Saúde da Família (USF) do Distrito Sanitário da Liberdade (DSL), caracterizando o perfil de utilização e identificando fatores relacionados à acessibilidade sócio-organizacional e geográfica. Métodos: Estudo transversal de base populacional com indivíduos de idade entre $20 e$ 75 anos, residentes da área de abrangência de duas USF. Analisou-se a acessibilidade segundo a procura ao serviço de saúde, a partir das características sócio-organizacional e geográfica e a utilização para consultas médicas na USF de referência. Resultados: Mais de 50\% dos entrevistados procuraram o serviço das USF e destes $78,7 \%$ afirmaram ter utilizado a consulta. Em relação à acessibilidade organizacional encontraram-se problemas como longo tempo de espera pela consulta. Discussão: Barreiras geográficas foram encontradas no processo de utilização da USF, contudo estas não impediram a realização da consulta. A utilização da USF local foi maior entre as pessoas com menor nivel socioeconômico.

Palavras-chave Acessibilidade a serviços de saúde, Utilização, Estratégia Saúde da Família 


\section{Introdução}

Acessibilidade a serviços de saúde refere-se às características destes que permitem que sejam facilmente utilizados pelos usuários potenciais ${ }^{1}$, uma temática importante da avaliação em saúde, por tratar da disponibilidade e distribuição social destes recursos ${ }^{2}$.

Em todo o mundo, a persistência de barreiras no processo de busca e utilização dos serviços gera oportunidades diferenciadas entre os grupos sociais na obtenção do cuidado em saúde, que muitas vezes caracterizam situações de injustiça soci$\mathrm{al}^{2}$. Ou seja, indivíduos com condições socioeconômicas mais favoráveis fazem maior e melhor uso dos serviços de saúde e a redução dessas desigualdades sociais é um desafio atual, tanto nos países industrializados e desenvolvidos, como em países dependentes e periféricos, independente do modelo de atenção à saúde ${ }^{3-7}$.

No Brasil, estudos sobre a temática, conduzidos desde a década de 1980, têm evidenciado tanto desigualdades marcantes no processo de utilização de serviços de saúde entre as diferentes regiões, estados e no interior dos municípios ${ }^{2,8,9}$, como demonstrado avanços resultantes de iniciativas de organização voltadas para a melhoria do acesso e acolhimento, que tendem a reduzir os obstáculos e barreiras e a facilitar a utilização dos mesmos pelos usuários ${ }^{10-12}$.

Outros estudos, apesar de reconhecerem a ampliação da oferta de serviços em municípios, revelam a persistência de problemas relacionados ao modo de organização, como a existência de barreiras decorrentes dos horários de funcionamento das unidades e dificuldade de atendimento à demanda espontânea ${ }^{11,13,14}$. Um desses trabalhos, realizado em município da Bahia em gestão plena, identificou como principais fatores dificultadores da utilização dos serviços de saúde os modelos de marcação de consultas, a falta de organização da referência para serviços especializados e a ausência de ações voltadas para o acolhimento ${ }^{11}$.

O Programa Saúde da Família (PSF) representa um importante instrumento para a reorganização do modelo assistencial, inclusive pelo seu potencial para reduzir as desigualdades sociais existentes na utilização de serviços ${ }^{10}$. O PSF oferta à população ações e recursos de saúde de forma mais adequada que as unidades básicas de saúde focado em ações programáticas, atividades domiciliares e, articulação com a comunidade $^{15}$, resultando em uma avaliação positiva por parte dos usuários quando comparado ao mo- mento anterior da implantação, em que a oferta de serviço de saúde era muito restrita e de baixa qualidade $^{16}$.

Dois estudos realizados em capitais brasileiras apresentaram evidências que apoiaram a hipótese do papel do PSF de promotor de equida$\mathrm{de}^{17,18}$, enquanto um terceiro revelou problemas de acessibilidade geográfica ${ }^{17}$. O primeiro, em áreas de abrangência de equipes de saúde da família em Porto Alegre, demonstrou que a utilização desse serviço foi maior entre a população com menor nível socioeconômico e sem cobertura por plano de saúde ${ }^{17}$, e o segundo, realizado em São Paulo, comparando áreas cobertas e não cobertas pelo PSF, observou que, enquanto nas áreas não cobertas houve uma maior utilização de serviços entre os grupos mais privilegiados, com maior poder aquisitivo e maior grau de instrução, nas áreas cobertas não foram verificadas diferenças de utilização segundo as variáveis renda e escolaridade ${ }^{18}$. O terceiro, uma avaliação de acessibilidade realizada em um distrito sanitário de Salvador, evidenciou que as barreiras geográficas, principalmente ladeiras, escadarias, alagamentos e custo com transporte, se constituíram em fatores limitantes para o acesso e a utilização de serviços em unidades de saúde da família ${ }^{19}$.

Diversos estudos ressaltam a importância de promover melhorias na organização dos serviços de saúde voltados para a garantia da acessibilidade e a integralidade do cuidado ${ }^{16,20} \mathrm{e}$ os resultados obtidos de pesquisa sobre a utilização de serviços de saúde e seus determinantes são de grande relevância pela possibilidade de orientar a formulação de políticas públicas que objetivem equidade na utilização destes serviços, bem como avaliação dos seus efeitos ${ }^{2,8,21}$.

Diante da importância da atenção básica como porta de entrada ao sistema de saúde e da potencialidade do PSF na reorganização das ações, este trabalho pretende caracterizar a acessibilidade e a utilização de serviços de saúde em duas unidades de saúde da família do Distrito Sanitário da Liberdade (DSL), no período de 2009-2010, caracterizando o perfil de utilização e identificando fatores relacionados à acessibilidade sócioorganizacional e geográfica.

\section{Metodologia}

Este estudo faz parte do Projeto "Acessibilidade às ações de atenção primária e adoção de hábitos saudáveis em populações cobertas pelo PACS/ PSF"22, desenvolvido pelo Instituto de Saúde 
Coletiva, da Universidade Federal da Bahia, em 2010. Trata-se de um inquérito domiciliar com indivíduos residentes em áreas cobertas pelo PSF, no Distrito Sanitário da Liberdade da cidade de Salvador, Bahia.

O Distrito analisado apresenta um território de $6,65 \mathrm{~km}^{2}$, com uma população estimada em 168.810 habitantes $^{23}$. A rede de serviços públicos de atenção à saúde conta com três unidades básicas de saúde (UBS), duas unidades de saúde da família (USF), uma Unidade de Atendimento Odontológico (UAO), um Centro de Atenção Psicossocial - infância e adolescente (CAPS-ia), seis unidades hospitalares sendo uma federal, e duas de emergência sob gestão estadual.

A população do estudo foi composta por indivíduos com idade entre 20 e 75 anos, residentes da área de abrangência das duas unidades de saúde da família (USF) do distrito pesquisado. A primeira unidade com duas equipes, com 9.396 pessoas cadastradas sendo 2.243 famílias e a segunda unidade com quatro equipes, com 15.235 pessoas cadastradas e 4.366 famílias $^{23}$.

Foi obtida uma amostra aleatória e representativa da população residente na área de abrangência das ESF em dois estágios. No primeiro foram sorteadas 1500 famílias para cada uma das áreas de abrangência das equipes, a partir das fichas de cadastramento familiar (ficha A) do Sistema de Informação da Atenção Básica (SIAB). A primeira família de cada área foi sorteada entre as dez primeiras da lista de cadastro usando a numeração estabelecida no SIAB. O intervalo para a seleção das demais famílias foi calculado para cada área em função do número total existente, considerando a cota de 250 por área. A partir do ponto inicial, essas foram incluídas, usando-se o intervalo calculado, até completar a amostra definida para cada área. Foram realizadas substituições das famílias, quando não havia membros destas dentro dos critérios de inclusão ou quando o domicílio estava fechado. Utilizou-se como critério para primeira substituição a família seguinte à sorteada no cadastro do SIAB, e para a segunda substituição, a família antecessora à substituída no cadastro do SIAB.

No segundo estágio, para a seleção do entrevistado , procedeu-se o sorteio de um indivíduo na faixa etária definida em cada família. Nesta etapa, na impossibilidade de se realizar a entrevista com o sorteado, marcava-se o retorno, que poderiam ser no máximo de três, caso contrário, a família seria excluída, não sendo substituída.

A coleta de dados foi conduzida entre dezembro de 2009 a fevereiro de 2010 por entrevistado- res devidamente treinados. Os dados foram coletados nos domicílios através de entrevista estruturada e pré-codificada que apresentava duas partes: a primeira, com as informações sobre todos os residentes do domicílio; e a segunda, com informações apenas do indivíduo sorteado.

Para fins desse estudo, os desfechos analisados foram "procura" e "utilização" de serviços de consulta nas unidades de saúde da família. Considerou-se consulta nesse estudo o atendimento realizado por profissional de nível superior (médico, enfermeiro ou odontólogo), a partir das perguntas: No último ano, o (a) Sr (a) procurou os serviços da Unidade Saúde da Família de referência? O (a) Sr. (a) passou por alguma consulta nesta Unidade no último ano? Por que prefere ser atendido na USF de referência?

A análise da acessibilidade considerou duas dimensões: a sócio-organizacional e a geográfica. Quanto os aspectos organizacionais, foram observadas características de funcionamento como: tempo de agendamento da consulta, horário de funcionamento do serviço, escuta dos profissionais da recepção, escuta dos profissionais de nível superior e identificar o nome do ACS.

A acessibilidade geográfica, por sua vez, foi avaliada através do tempo de deslocamento a pé da residência do usuário até a USF, uso de transporte para o deslocamento da residência à USF de referência e obstáculos existentes no processo de busca pelo serviço.

Para traçar o perfil da população que utilizou e que não utilizou as unidades de saúde da família, foram empregadas as variáveis independentes sexo, idade e raça (cor da pele), ocupação, escolaridade, cobertura por planos de saúde, local de residência, autopercepção do estado de saúde.

Os dados foram processados com o auxílio dos softwares Epiinfo 6.0 e STATA 10, sendo realizados a análise descritiva (frequência absoluta e relativa) e o teste qui-quadrado de Pearson, com nível de significância de 0,05 .

O projeto foi aprovado pelo Comitê de Ética em Pesquisa do Instituto de Saúde Coletiva da Universidade Federal da Bahia. Os entrevistados foram orientados sobre a pesquisa e assinaram o Termo de Consentimento Livre e Esclarecido, conforme a Resolução 196/96 ${ }^{24}$ do Conselho Nacional de Saúde.

\section{Resultados}

Do total de entrevistados, 670 (56\%) procuraram a sua unidade de saúde da família de refe- 
rência nos 12 meses que antecederam a pesquisa e destes, $527(78,7 \%)$ afirmaram ter realizado consulta por profissional de nível superior na unidade neste período.

Em relação às características sociodemográficas dos entrevistados, pode-se observar que a USF de referência foi procurada em maior proporção pelos adultos acima dos 60 anos (59,9\%). Com relação ao sexo, a busca pelo serviço foi maior entre as mulheres $(61,3 \%)$ do que entre os homens $(42,4 \%)$, quanto à raça, a procura foi maior entre os brancos $(60,5 \%)$ (Tabela 1$)$.

Quando se compara o perfil dos usuários que utilizaram as USF de referência no último ano com aqueles que não utilizaram, percebe-se que houve diferença significativa na utilização pelo sexo e o fato de possuir plano de saúde. Entre as mulheres,
$82,3 \%$ utilizaram o serviço de saúde enquanto entre os homens o uso foi de $64,5 \%$ e entre aqueles que possuem plano de saúde, o uso foi de $62 \%$ ao passo que, entre os que não possuem $80,4 \%$ realizaram consulta na unidade (Tabela1).

Nas variáveis idade, raça, ocupação e autopercepção de saúde não houve diferença nas distribuições. Vale destacar que entre os entrevistados que referiram a procura pela unidade de saúde de referência, mas que não realizaram a consulta, foi possível observar maior proporção de indivíduos do sexo masculino (35,5\%), com mais de oito anos de estudo $(24,5 \%)$ e com planos de saúde $(38,0 \%)$ (Tabela1).

Ressalta-se que para algumas variáveis houve algumas perdas no número absoluto das respostas, o que justifica diferenças no número total $(\mathrm{N})$.

Tabela 1. Características socioeconômicas, demográficas e de saúde dos usuários que procuraram, utilizaram e não utilizaram a unidade de referência no Distrito Sanitário da Liberdade de Salvador (BA), 2010.

\begin{tabular}{|c|c|c|c|c|c|}
\hline & \multicolumn{2}{|c|}{ Procuraram } & \multirow{2}{*}{$\begin{array}{c}\text { Utilizaram } \\
\%\end{array}$} & \multirow{2}{*}{$\frac{\text { Não utilizaram }}{\%}$} & \multirow[b]{2}{*}{$\mathbf{P}^{\star}$} \\
\hline & $\mathbf{n}$ & $\%$ & & & \\
\hline \multicolumn{6}{|l|}{ Idade } \\
\hline Adulto Jovem $<40$ & 294 & 56,1 & 75,8 & 24,2 & \\
\hline Meia-idade $\geq 40$ a $<60$ & 257 & 54,2 & 80,0 & 20,0 & 0,280 \\
\hline Terceira-idade $\geq 60$ & 121 & 59,9 & 82,0 & 18,0 & \\
\hline \multicolumn{6}{|l|}{ Sexo } \\
\hline Masculino & 142 & 42,4 & 64,5 & 35,5 & \\
\hline Feminino & 530 & 61,3 & 82,3 & 17,7 & 0,000 \\
\hline \multicolumn{6}{|l|}{ Raça } \\
\hline Branco & 49 & 60,5 & 80,0 & 20,0 & \\
\hline Negro & 332 & 53,7 & 80,0 & 20,0 & 0,607 \\
\hline Pardo & 274 & 57,1 & 77,7 & 22,3 & \\
\hline Outros & 42 & 62,7 & 71,4 & 28,6 & \\
\hline \multicolumn{6}{|l|}{ Escolaridade } \\
\hline Analfabeto & 31 & 56,4 & 83,9 & 16,1 & \\
\hline 1 a 8 anos de estudo & 228 & 61,0 & 83,3 & 16,7 & 0,052 \\
\hline Mais de 8 anos de estudo & 413 & 53,6 & 75,5 & 24,5 & \\
\hline \multicolumn{6}{|l|}{ Ocupação } \\
\hline Desempregado (a procura de trabalho) & 133 & 64,6 & 78,8 & 21,2 & \\
\hline Estudante & 15 & 42,9 & 80,0 & 20,0 & 0,870 \\
\hline Responsável apenas pelo cuidado da casa & 193 & 65,4 & 80,4 & 19,6 & \\
\hline Sem ocupação no momento & 57 & 53,3 & 80,7 & 19,3 & \\
\hline Outro & 269 & 49,0 & 76,5 & 23,5 & \\
\hline \multicolumn{6}{|l|}{ Plano de saúde } \\
\hline Possui & 72 & 39,3 & 62,0 & 38,0 & \\
\hline Não possui & 597 & 59,0 & 80,4 & 19,6 & 0,000 \\
\hline \multicolumn{6}{|l|}{ Auto percepção da Saúde } \\
\hline Muito boa & 43 & 38,7 & 65,1 & 34,9 & \\
\hline Boa & 276 & 51,7 & 77,9 & 22,1 & 0,067 \\
\hline Regular/Ruim & 349 & 64,0 & 80,5 & 19,5 & \\
\hline Total & 672 & 100,0 & 78,5 & 21,5 & \\
\hline
\end{tabular}

${ }^{\star}$ Intervalo com 95 de confiança. 
Quanto à procura pela USF de Referência no último ano, esta foi superior a $50 \%$ em 5 das equipes analisadas, entretanto, nenhuma alcançou 70\% da procura. Ambas as equipes da USF 1 apresentaram os menores percentuais de procura da unidade. Vale destacar, no entanto, que essa unidade foi referida pelos entrevistados como a que possui o menor tempo de deslocamento a pé entre as esquipes analisadas (Tabela 2).

Em média o tempo de deslocamento a pé até a unidade de referência foi menor que dez minutos para cerca de $50 \%$ dos entrevistados, o que justifica o baixo percentual em relação ao uso de transporte nessas áreas, tendo a equipe 1 da USF 10 maior percentual de pessoas que demoram menos de 10 minutos no deslocamento entre a residência e a USF. Já na USF2 (equipes 3, 4 e 6) foi maior o percentual de pessoas que o tempo de deslocamento supera a 20 minutos (Tabela 2).

Embora as barreiras geográficas tenham sido identificadas como obstáculos para a utilização da consulta nas Unidades de Saúde da Família de referência, estas não se mostraram como impedimentos à utilização da consulta nas duas USF investigadas. Assim, 79,2\% dos entrevistados que apontaram algum tipo de barreira geográfica fizeram uso da consulta nas Unidades de Saúde da Família de referência (Tabela 3).

O tempo de agendamento constitui-se como uma barreira evidente para os usuários que procuraram pela USF de referência. Em todas as unidades verificou-se que $68,8 \%$ ou mais dos usuários referiram tempo superior a 7 dias entre o agendamento e a consulta. (Tabela 4)

Como facilitadores para o acesso ao serviço de referência o horário de funcionamento da USF de Referência (83,7\%), a escuta pelos profissionais da recepção $(76,9 \%)$ e a escuta pelos profissionais de nível superior foram referidas por uma parte significativa dos entrevistados. Ressalta-se a equipe 2 (USF 1) na qual 75,2\% dos entrevistados que procuraram o serviço identificaram o nome do ACS que realizam o acompanhamento de suas famílias (Tabela 4).

A equipe 5 (USF 2) obteve os maiores percentuais de entrevistados que referiram a escuta dos profissionais da recepção $(81,3 \%)$ e dos profissionais do nível superior $(88,1 \%)$, bem como o reconhecimento do nome do ACS que acompanham suas famílias $(75,1 \%)$ (Tabela 4$)$.

As razões para a procura da USF de referência entre aqueles que utilizaram foram a proximidade geográfica $(45,7 \%)$, a qualidade do atendimento $(28,1 \%)$ e o menor tempo para acessar o serviço $(7,5 \%)$. Vale destacar que outros moti-

Tabela 3. Número de pessoas que utilizaram e que não utilizaram a consulta na USF de referência no último ano segundo obstáculos identificados no deslocamento, Distrito Sanitário da Liberdade, Salvador-Ba, 2010.

\begin{tabular}{lccc}
\hline & $\begin{array}{c}\text { Total } \\
\mathbf{n}\end{array}$ & $\begin{array}{c}\text { Utilizou } \\
\text { \% }\end{array}$ & $\begin{array}{c}\text { Não utilizou } \\
\%\end{array}$ \\
\hline Ladeira & 254 & 79,5 & 20,5 \\
Escadaria & 197 & 80,7 & 19,3 \\
Avenida com grande tráfego & 431 & 78,6 & 21,4 \\
Córrego & 402 & 78,6 & 21,4 \\
Área de alagamento & 449 & 78,6 & 21,4 \\
Outro & 19 & 79,0 & 21,0 \\
Total & 1752 & 79,2 & 20,8 \\
& & &
\end{tabular}

Tabela 2. Características da acessibilidade geográfica no processo de procura pelas USFs do Distrito Sanitário da Liberdade por área de abrangência - Salvador (BA), 2010.

\begin{tabular}{|c|c|c|c|c|c|c|c|}
\hline & \multirow[b]{2}{*}{ Total } & \multirow{2}{*}{$\begin{array}{c}\text { Procuraram a } \\
\text { USF referência } \\
\%\end{array}$} & \multirow{2}{*}{$\begin{array}{c}\text { Outro serviço } \\
\text { de saúde } \\
\%\end{array}$} & \multicolumn{3}{|c|}{$\begin{array}{l}\text { Tempo de deslocamento a pé } \\
\text { da casa à USF }\end{array}$} & \multirow{2}{*}{$\begin{array}{c}\text { Uso de transporte } \\
\text { para a USF } \\
\%\end{array}$} \\
\hline & & & & $\begin{array}{c}\leq 10 \\
\%\end{array}$ & $\begin{array}{c}>10 \mathrm{e} \leq 20 \\
\%\end{array}$ & $\begin{array}{c}>20 \min \\
\%\end{array}$ & \\
\hline \multicolumn{8}{|l|}{ USF 1} \\
\hline Equipe 1 & 171 & 50,3 & 49,7 & 87,8 & 11,3 & 0,9 & 0,9 \\
\hline Equipe 2 & 217 & 46,8 & 53,2 & 52,8 & 39,2 & 8,0 & 5,7 \\
\hline \multicolumn{8}{|l|}{ USF2 } \\
\hline Equipe 3 & 215 & 61,5 & 38,5 & 47,0 & 35,0 & 18,0 & 8,5 \\
\hline Equipe 4 & 229 & 65,1 & 34,9 & 33,1 & 39,4 & 27,6 & 3,9 \\
\hline Equipe 5 & 164 & 52,7 & 47,3 & 56,0 & 36,3 & 7,7 & 3,3 \\
\hline Equipe 6 & 204 & 67,5 & 32,5 & 22,0 & 44,0 & 34,1 & 4,4 \\
\hline Total & 1200 & 57,7 & 42,3 & 50,3 & 33,9 & 15,8 & 4,5 \\
\hline
\end{tabular}


vos também foram apontados pelos usuários $(18,7 \%)$, entre eles destacam-se: orientação do ACS, facilidade de marcação, oferta de exames e serviço de emergência/ urgência (Tabela 5).

\section{Discussão}

Os serviços das USF de referência foram procurados no último ano por mais da metade dos entrevistados, demonstrando avanços importantes quanto à conformação da Estratégia de Saúde da Família como primeiro contato ou porta de entrada prioritária do sistema de saúde.

A utilização da consulta na USF de referência no presente estudo foi superior ao encontrado em outros como, por exemplo, na investigação reali- zada em Porto Alegre em áreas cobertas pelo PSF, na qual se identificou uma prevalência de utilização da consulta médica nos 15 dias anteriores à entrevista de $13,5 \%{ }^{17}$. A elevada utilização verificada no presente estudo está de acordo com as evidências de outras experiências municipais que revelam que a expansão da oferta e a reorganização dos serviços de saúde, ocorridos nos últimos vinte anos, e, sobretudo, a partir da definição do PSF como porta de entrada preferencial ao sistema de saúde, tem viabilizado maior utilização dos serviços de saúde desse nível de atenção ${ }^{17,25-32}$.

Ainda que tenham sido observadas semelhanças entre o perfil da população que utilizou a consulta no último ano e aquela que não o fez, cabe destacar que a ausência de planos de saúde e estar desempregado foram aspectos que con-

Tabela 4. Características organizacionais da acessibilidade no processo de procura aos serviços de saúde na área de abrangência das USFs de referência do Distrito Sanitário da Liberdade - Salvador (BA), 2010.

\begin{tabular}{|c|c|c|c|c|c|c|c|}
\hline & \multirow{2}{*}{ Total } & \multicolumn{2}{|c|}{$\begin{array}{c}\text { Tempo de } \\
\text { agendamento }\end{array}$} & \multirow{2}{*}{$\begin{array}{c}\text { Horário de } \\
\text { funcionamento } \\
\%\end{array}$} & \multirow{2}{*}{$\begin{array}{c}\text { Escuta dos } \\
\text { profissionais } \\
\text { da recepção } \\
\% \\
\end{array}$} & \multirow{2}{*}{$\begin{array}{c}\text { Escuta dos } \\
\text { profissionais de } \\
\text { nível superior } \\
\%\end{array}$} & \multirow{2}{*}{$\begin{array}{c}\text { Conhece o } \\
\text { nome do } \\
\text { ACS } \\
\%\end{array}$} \\
\hline & & $\begin{array}{c}\leq 7 \text { dias } \\
\%\end{array}$ & $\begin{array}{c}>7 \text { dias } \\
\%\end{array}$ & & & & \\
\hline \multicolumn{8}{|l|}{ USF 1} \\
\hline Equipe 1 & 171 & 27,6 & 72,4 & 77,4 & 72,8 & 79,5 & 70,8 \\
\hline Equipe 2 & 217 & 21,6 & 78,4 & 78,4 & 79,7 & 73,8 & 75,2 \\
\hline \multicolumn{8}{|l|}{ USF2 } \\
\hline Equipe 3 & 215 & 21,9 & 78,1 & 91,5 & 73,9 & 87,8 & 60,2 \\
\hline Equipe 4 & 229 & 27,4 & 72,6 & 90,7 & 75,0 & 83,6 & 66,4 \\
\hline Equipe 5 & 164 & 28,2 & 71,8 & 82,6 & 81,3 & 88,1 & 75,1 \\
\hline Equipe 6 & 204 & 31,3 & 68,8 & 80,2 & 80,4 & 75,3 & 67,5 \\
\hline Total & 1200 & 26,0 & 74,0 & 83,7 & 76,9 & 81,2 & 68,9 \\
\hline
\end{tabular}

Tabela 5. Usuários que utilizaram a USF de referência no último ano e os principais motivos para utilização dessas Unidades, Distrito Sanitário da Liberdade, Salvador-Ba, 2010.

\begin{tabular}{cccccc}
\hline & Total & $\begin{array}{c}\text { Proximidade } \\
\text { geográfica } \\
\%\end{array}$ & $\begin{array}{c}\text { Menor tempo para } \\
\text { acessar o serviço } \\
\%\end{array}$ & $\begin{array}{c}\text { Qualidade do } \\
\text { atendimento } \\
\%\end{array}$ & $\begin{array}{c}\text { Outro motivo } \\
\%\end{array}$ \\
\hline USF 1 & & & & & \\
Equipe 1 & 74 & 12,4 & 18,2 & 12,2 & 15,6 \\
Equipe 2 & 107 & 23,6 & 31,8 & 23,2 & 12,8 \\
USF2 & & & & & \\
Equipe 3 & 91 & 18,7 & 11,4 & 18,3 & 17,4 \\
Equipe 4 & 110 & 22,5 & 20,5 & 20,7 & 11,9 \\
Equipe 5 & 72 & 14,6 & 11,4 & 8,1 & 33,0 \\
Equipe 6 & 73 & 8,2 & 6,8 & 28,1 & 18,7 \\
Total & 527 & 45,7 & 7,5 & &
\end{tabular}


tribuíram para maior utilização da USF de referência entre os entrevistados. Desta forma, observa-se que o Programa de Saúde da Família pode estar contribuindo para a redução das desigualdades e iniquidades em relação à utilização de serviços de saúde no território analisado. Situação semelhante foi identificada em áreas cobertas pela ESF nos estudos de Fernandes et al. ${ }^{17}$ e Goldbaun et al. ${ }^{18}$. Destaca-se ainda que o acesso aos cuidados de saúde não pode ser discutido de forma isolada de outros problemas existentes como o desemprego e a pobreza generalizada, pois os mesmos o afetam ${ }^{33}$.

De maneira geral, os serviços de saúde são melhores e mais utilizados pelos indivíduos com melhores condições socioeconômicas ${ }^{2,8,21,34-39}$. No entanto, nesse estudo, os indivíduos que não apresentavam cobertura por plano de saúde, analfabetos e sem ocupação no momento da entrevista utilizaram mais o serviço de consulta nas USF de referência.

Ao analisar o fator idade observou-se uma maior utilização da consulta na USF de referência entre a população idosa, entretanto essa variável não apresentou significância estatística. Diversos autores ${ }^{17,22,26,35,40,41}$ também observaram maior utilização da consulta entre a população com mais idade, fato que poderia estar associado à prevalência de doenças crônicas não transmissíveis nesse grupo etário ${ }^{34}$.

Observou-se neste estudo a persistência de problemas tradicionais relacionados à acessibilidade organizacional, como o longo tempo de espera para realização da consulta, fato este que pode estar contribuindo para afastar o paciente da procura pelo serviço de saúde. Situação semelhante foi identificada em estudo realizado em um município do interior da Bahia, no qual a acessibilidade aliada à ausência de marcação permanente de consulta tende a dificultar o acesso ${ }^{11}$.

Fatores como horário de funcionamento, escuta pelos profissionais da recepção e pelos de nível superior foram referidos como facilitadores para o acesso aos serviços de saúde. Schimith e Lima ${ }^{42}$, em estudo qualitativo sobre o trabalho realizado em uma ESF, evidenciaram a importância do encontro entre usuários e profissionais e do estabelecimento da escuta para a identificação das necessidades de saúde trazidas por aquelas pessoas que buscam o serviço.

O ACS apareceu como importante elo entre a população e a USF, facilitando o acesso aos serviços, fato que pode ser constatado pelo elevado número de usuários que identificaram o nome do ACS responsável pelo acompanhamento de suas famílias. Estudo de Nunes et al. ${ }^{43}$ também evidenciou a presença do ACS como importante mediador entre a comunidade e os profissionais de saúde, facilitando o acesso ao cuidado.

A existência de barreiras geográficas foi percebida no processo de deslocamento até a USF de referência por uma parte significativa dos entrevistados, situação referida em diversos estudos que apontam fatores geográficos como barreiras para utilização dos serviços de saúde ${ }^{33,44-47}$. Em outro estudo realizado anteriormente nesse DS em áreas cobertas pelo Programa de Agentes Comunitários de Saúde (PACS) e Programa de Saúde da Família (PSF), as barreiras geográficas (ladeiras, escadarias, alagamentos, custo com transporte) foram apontadas como importantes obstáculos para o acesso aos serviços de saú$\mathrm{de}^{29}$. Ainda no referido estudo, ficou evidente que o processo de implantação da estratégia PACS/ PSF e o modelo de territorialização adotado neste Distrito não atenderam aos princípios que devem nortear a organização da atenção primária à saúde, o que representou restrições na acessibilidade geográfica da população coberta nessa área.

Vale destacar que embora as barreiras geográficas tenham sido identificadas como obstáculos para a utilização da consulta nas Unidades de Saúde da Família de referência, estas não impediram a utilização da consulta nas duas USF pesquisadas. Assim, pode-se inferir que outros fatores, como a necessidade de saúde e o modo de organização do serviço podem contribuir para uma maior utilização na população entrevistada. Diversos estudos evidenciaram importante associação entre a utilização dos serviços e a percepção de saúde ${ }^{8,17,20,27}$. Para Andersen e Newman ${ }^{5}$, por exemplo, a autopercepção de saúde representa a causa mais imediata do uso dos serviços.

Problemas como a concentração de mais de quatro equipes de saúde da família em uma única unidade com o objetivo de garantir a cobertura de grandes extensões territoriais pode provocar problemas de acessibilidade geográfica. Assim, torna-se necessário refletir sobre a expansão da ESF, com a presença de duas ou três equipes por $\mathrm{USF}^{19}$, com o objetivo de garantir uma maior eficiência nas ações.

No entanto, vale destacar que apesar da identificação de algumas barreiras para a procura e utilização das USF analisadas, o acesso à ESF vem contribuindo para a organização do sistema de saúde, como porta de entrada importante no distrito pesquisado e para a equidade, minimizando desigualdades sociais na utilização dos serviços de saúde no território pesquisado. 
As informações obtidas com este estudo, a partir da percepção dos usuários sobre a acessibilidade geográfica e organizacional às USF, apesar de algumas limitações, como o longo período recordatório, se mostram de grande relevância para o planejamento e o aprimoramento dos serviços de saúde no distrito pesquisado. Outros estudos deverão ser realizados no sentido de identificar a qualidade do atendimento oferecido nas Unidades de Saúde da Família pesquisadas.

A elaboração de projetos que fortaleçam a educação em serviço e o desenvolvimento de manuais e rotinas para atenção básica, com o propósito de fortalecer a humanização do cuidado atra- vés de práticas de acolhimento e a consolidação da proposta da ESF de mudança do modelo assistencial, somado a ações intersetoriais, favorece a ampliação da acessibilidade organizacional.

A partir deste estudo pôde-se perceber que a utilização dos serviços de saúde resulta da interação complexa de fatores individuais, sociais e organizacionais, como já identificado por outros autores ${ }^{2,17,18,20,21}$. Os achados deste trabalho apontaram para a necessidade de se conhecer melhor os usuários e os fatores que interferem no uso dos serviços bem como nas medidas necessárias para que os serviços de saúde se adéquem à diversidade existente entre eles.

\section{Colaboradores}

LS Oliveira, LG Nascimento, MAS Oliveira e GB Gil trabalharam na concepção, análise, interpretação dos dados, redação do artigo. ABO Cunha trabalhou no delineamento do estudo, redação e revisão do artigo, MG Medina e RAG Pereira no delineamento do estudo, revisão do artigo e aprovação da versão a ser publicada. 


\section{Referências}

1. Donabedian A. Los espacios de la salud: aspectos fundamentales de la organización de la atención médica. México DF: Editora Biblioteca de la Salud; 1988.

2. Travassos C, Castro MSM. Determinantes e desigualdades sociais no acesso e na utilização dos serviços de saúde. In: Giovanella L. Políticas e sistema de saúde no Brasil. Rio de Janeiro: Fiocruz, Cebes; 2008. p. 215-243.

3. Field KS, Briggs DJ. Socio-economic and locational determinants of accessibility and utilization of primary health-care. Health Soc Care Community 2001; 9(5):294-308.

4. Wilson K, Rosemberg MW. Accessibility and the Canadian health care system: squaring perceptions and realities. Health Policy 2004; 67(2):137-148

5. Andersen R, Newman JF. Societal and Individual Determinants of Medical Care Utilization in the United States. Milbank Mem Fund Q Health Soc 1973; 51(1):95-124.

6. La Hoz KF, Leon DA. Self-perceived health status and inequalities in use of health services in Spain. Int J Epidemiol 1996; 25(3):593-603.

7. Travassos C, Oliveira EXG, Viacava F. Desigualdades geográficas e sociais no acesso aos serviços de saúde no Brasil: 1998 e 2003. Cien Saude Colet 2006; 11(4):975-986.

8. Sawyer DO, Leite IC, Alexandrino R. Perfis de utilização de serviços de saúde no Brasil. Cien Saude Colet 2002; 7(4):757-766.

9. Travassos C, Viacava F, Fernandes C, Almeida C. Desigualdades geográficas e sociais na utilização de serviços de saúde no Brasil. Cien Saude Colet 2000; 5(1):133-149.

10. Senna MCM. Equidade e política de saúde: algumas reflexões sobre o programa saúda da família. Cad Saude Publica 2002; 18(Supl. 1):S203-S2011.

11. Cunha ABO, Vieira-da-Silva LM. Acessibilidade aos serviços de saúde em um município do Estado da Bahia, Brasil, em gestão plena do sistema. Cad Saude Publica 2010; 26(4):725-737.

12. Vieira-da-Silva LM, Hartz ZMA, Chave SCL, Silva GAP, Paim JS. Análise da implantação da gestão descentralizada em saúde: estudo comparado de cinco casos na Bahia, Brasil. Cad Saude Publica 2007; 23(2):355-370

13. Escorel S, Giovanella L, Mendonça MHM, Senna MCM. O Programa de Saúde da Família e a construção de um novo modelo para a atenção básica no Brasil. Rev Panam Salud Publica 2007; 21 (2 3):164-176

14. Trad LAB, Bastos ACS, Santana EM, Nunes MO. Estudo etnográfico da satisfação do usuário do Programa de Saúde da Família (PSF) na Bahia. Cien Saude Colet 2002; 7(3):581-589.

15. Facchini LA, Piccini RA, Tomasi E, Thumé E, Silveira DS, Siqueira FV, Rodrigues AR. Desempenho do PSF no sul e no nordeste do Brasil: avaliação institucional e epidemiológica da atenção básica à saúde. Cien Saude Colet 2006; 11(3):669-681.
16. Schwartz TD, Ferreira JTB, Maciel ELN, Lima RCD. Estratégia Saúde da Família: avaliando o acesso ao SUS a partir da percepção dos usuários da Unidade de Saúde de Resistência, na região de São Pedro, no município de Vitória (ES). Cien Saude Colet 2010; 15(4):2145-2154.

17. Fernandes LCL, Bertoldi AD, Barros AJD. Utilização dos serviços de saúde pela população coberta pela Estratégia de Saúde da Família. Rev Saude Publica 2009; 43(4):595-603.

18. Goldbaum M, Gianini RJ, Novaes, HMD, Cesar CLG. Utilização de serviços de saúde em áreas cobertas pelo programa saúde da família (Qualis) no Município de São Paulo. Rev Saude Publica 2005; 39(1):90-99.

19. Silva Júnior ES, Medina MG, Aquino R, Fonseca ACF, Vilasbôas ALQ. Acessibilidade geográfica à atenção primária à saúde em distrito sanitário do município de Salvador, Bahia. Rev Bras Saude Mater Infant 2010; 10(Supl. 1):S49-S60.

20. Louvison MCP, Lebrão ML, Duarte YAO, Santos JLF, Malik AM, Almeida ES. Desigualdades no uso e acesso aos serviços de saúde entre idosos do município de São Paulo. Rev Saude Publica 2008; 42(4):733-740.

21. Travassos C, Viacava F, Fernandes C, Almeida C. Desigualdades geográficas e sociais na utilização de serviços de saúde no Brasil. Cien Saude Colet 2000; 5(1):133-149.

22. Medina MG, Aquino R. Promoção da saúde no âmbito da estratégia de saúde da família do Distrito Sanitário Liberdade [Projeto de Pesquisa e Cooperação Técnica]. Salvador: ISC, UFBA; 2011. (mimeo)

23. Salvador. Subcoordenação de Informação de Saúde, Secretaria Municipal de Saúde. [site na Internet]. [acessado 2012 abr 24]. Disponível em: http:/ /www.tabnet.saude.salvador.ba.gov.br/

24. Brasil. Ministério da Saúde. Conselho Nacional de Saúde. Resolução no. 196/1996. Diretrizes e Normas Regulamentadoras de Pesquisas Envolvendo Seres Humanos. Diário Oficial da União 1996; out 10.

25. Travassos C, Viacava F, Fernandes C, Almeida C. Desigualdades geográficas e sociais na utilização de serviços de saúde no Brasil. Cien Saude Colet 2000; 5(1):133-149.

26. Instituto Brasileiro de Geografia e Estatística (IBGE) Pesquisa Nacional por Amostragem de Domicílios 2008. Um Panorama da Saúde no Brasil. Acesso e utilização dos serviços de saúde e fatores de risco e proteção à saúde. Rio de Janeiro: IBGE; 2010.

27. Ribeiro MCSA, Barata RB, Almeida MF, Silva ZP. Perfil sociodemográfico e padrão de utilização de serviços de saúde para usuários e não-usuários do SUS - PNAD 2003. Cien Saude Colet 2006; 11(4):10111022.

28. Dias-da-Costa JS, Fachinni LA. Utilização de serviços ambulatoriais em Pelotas: onde a população consulta e com que freqüência. Rev Saude Publica 1997; 31(4):360-369. 
29. Sousa MF. O Programa Saúde da Família no Brasil: análise do acesso à atenção básica. Rev bras Enferm 2008; 61(2):153-158.

30. Giovanella L, Mendonça MHM, Almeida PF, Escorel S, Senna MCM, Fausto MCR, Delgado MM, Andrade CLT, Cunha MS, Martins MIC, Teixeira CP. Saúde da família: limites e possibilidades para uma abordagem integral de atenção primária à saúde no Brasil. Ciênc Saúde 2009; 14(3):783-794.

31. Bousquat A, Alves MCGP, Elias PE. Utilização do Programa de Saúde da Família em regiões metropolitanas: abordagem metodológica. Rev Saude Publica 2008; 42(5):903-906.

32. Dias-da-Costa JS, Olinto MTA, Soares SA, Nunes MF, Bagatini T, Marques MC, Guimarães LK, Müller LP, Machado FCS, Barcellos ES, Pattussi MP. Utilização de serviços de saúde pela população adulta de São Leopoldo, Rio Grande do Sul, Brasil: resultados de um estudo transversal. Cad Saude Publica 2011; 27(5):868-876.

33. Rechel B, Blackburn CM, Spencer NJ, Rechel B. Access to health care for Roma children in Central and Eastern Europe: findings from a qualitative study in Bulgaria. Int J Equity Health 2009; 8:24

34. Mishima SM, Paula VG, Pereira MJB, Almeida MCP, Kawata LS. (In)Satisfação dos usuários: duplicação e uso simultâneo na utilização de serviços na Saúde da Família. Rev esc Enferm 2010; 44(3):766-773.

35. Travassos C, Viacava F, Pinheiro R, Brito A. Utilização dos serviços de saúde no Brasil: gênero, características familiares e condição social. Rev $\mathrm{Pa}$ nam Salud Publica 2002; 11(5-6):365-373.

36. Doorslaer E, Masseria C, Koolman X. Inequalities in access to medical care by income in developed countries. CMAJ 2006; 174(2):177-183

37. Godwin M, Hodgetts G, Bardon E, Seguin R, Packer D, Geddes J. Primary care in Bosnia and Herzegovina Health care and health status in general practice ambulatory care centers. Can Fam Physician 2001; 47:289-297.

38. PJ Veugelers, AM Yip. Socioeconomic disparities in health care use: Does universal coverage reduce inequalities in health? J Epidemiol Community $\mathrm{He}$ alth 2003; 57(6):424-428.
39. Rust G, Fryer GE Jr, Phillips RL Jr, Daniels E, Strothers H, Satcher D. Modifiable Determinants of Healthcare Utilization within the African-American Population. J Natl Med Assoc 2004; 96(9):1169-1177.

40. Dias-da-Costa JS, Olinto MTA, Gigante DP, Menezes AMB, Macedo S, Daltoé T, Santos IS, Fuchs SC. Utilização de serviços ambulatoriais de saúde em Pelotas, Rio Grande do Sul, Brasil: alguns fatores relacionados com as consultas médicas acima da média. Cad Saude Publica 2008; 24(2):353-363.

41. Capilheira MF, Santos IS. Fatores individuais associados à utilização de consultas médicas por adultos. Rev Saude Publica 2006; 40(3):436-443.

42. Schimith MD, Lima MADS. Acolhimento e vínculo em uma equipe do Programa Saúde da Família. Cad Saude Publica 2004; 20(6):1487-1494.

43. Nunes MO, Trad LB, Almeida BA, Homem CR, Melo MCIC. O agente comunitário de saúde: construção da identidade desse personagem híbrido e polifônico. Cad Saude Publica 2002; 18(6):1639-1646.

44. Ahluwalia S, Offredy M. A qualitative study of the impact of the implementation of advanced access in primary healthcare on the working lives of general practice staff. BMC Fam Pract 2005; 6:39

45. Sibley LM, Weiner JP. An evaluation of access to health care services along the rural-urban continuum in Canada. BMC Health Serv Res 2011; 11:20

46. Wallace SP, Gutiérrez VF. Equity of access to health care for older adults in four major Latin American cities. Rev Panam Salud Publica 2005; 17(5/6):394409.

47. Yiengprugsawan V, GA Carmichael, LL-Y Lim, S Seubsman and AC Sleigh. Explanation of inequality in utilization of ambulatory care before and after universal health insurance in Thailand. Health Policy Plan 2011; 26(2):105-114.

Artigo apresentado em 30/04/2012

Aprovado em 17/07/2012

Versão final apresentada em 30/08/2012 